\title{
Discrimination of thermal baths by single-qubit probes
}

\author{
Ilaria Gianani, ${ }^{1,2}$ Donato Farina $\odot,{ }^{3,4}$ Marco Barbieri, ${ }^{1,5}$ Valeria Cimini $\odot,{ }^{1}$ Vasco Cavina $\odot,{ }^{6}$ and Vittorio Giovannetti ${ }^{7}$ \\ ${ }^{1}$ Dipartimento di Scienze, Università degli Studi Roma Tre, Via della Vasca Navale 84, 00146 Rome, Italy \\ ${ }^{2}$ Dipartimento di Fisica, Sapienza Università di Roma, Piazzale Aldo Moro 5, 00185 Rome, Italy \\ ${ }^{3}$ NEST, Scuola Normale Superiore, 56126 Pisa, Italy \\ ${ }^{4}$ Graphene Labs, Istituto Italiano di Tecnologia, Via Morego 30, 16163 Genova, Italy \\ ${ }^{5}$ Istituto Nazionale di Ottica, CNR, Largo Enrico Fermi 6, 50125 Firenze, Italy \\ ${ }^{6}$ Complex Systems and Statistical Mechanics, Physics and Materials Science Research Unit, University of Luxembourg, \\ 1511 Luxembourg, Luxembourg \\ ${ }^{7}$ NEST, Scuola Normale Superiore and Istituto Nanoscienze, CNR, 56126 Pisa, Italy
}

(Received 6 May 2020; accepted 18 August 2020; published 25 September 2020)

\begin{abstract}
Nonequilibrium states of quantum systems in contact with thermal baths help distinguishing between environments with different temperatures or different statistics. We extend these studies to a more generic problem that consists in discriminating between two baths with disparate constituents at unequal temperatures. Notably there exist temperature regimes in which the presence of coherence in the initial state preparation is beneficial for the discrimination capability. We also find that nonequilibrium states are not universally optimal and detail the conditions in which it becomes advantageous to wait for complete thermalization of the probe. These concepts are illustrated in a linear-optical simulation.
\end{abstract}

DOI: 10.1103/PhysRevResearch.2.033497

\section{INTRODUCTION}

The reduced dynamics of a quantum system interacting with an external environment is typically insensitive to many characteristic features of the latter [1-3]. However, some macroscopic properties of the bath (say, its temperature) may have a nontrivial influence on the resulting equations of motion, paving the way for the possibility of probing these quantities via measurements performed on the system alone [4-14].

Relying on these observations, in Ref. [15] a statistics tagging scheme was presented, allowing the determination of the fermionic or bosonic character of a thermal bath $E$ by detecting the modifications induced on a quantum probing system $A$ put in thermal contact with $E$ for some proper interaction time $t$. The analysis was conducted assuming the temperature of the bath to be known and, most importantly, equal in the two alternative scenarios. Under this condition, waiting for the complete thermalization of $A$ (i.e., setting $t \rightarrow \infty$ ) is clearly not a viable option to get useful information on the nature of the bath. Indeed, as $t$ diverges the probe will be driven toward the same final thermal equilibrium configuration irrespectively of the statistics of $E$, hence keeping no track of its fermionic or bosonic character. As a consequence, the optimal discrimination performances in Ref. [15] were

Published by the American Physical Society under the terms of the Creative Commons Attribution 4.0 International license. Further distribution of this work must maintain attribution to the author(s) and the published article's title, journal citation, and DOI. obtained at times $t$ where the evolved state of $A$ was explicitly in a nonequilibrium condition. Superiority of nonequilibrium conditions for measurement purposes is not unique to the statistics tagging procedure discussed in [15]; similar behavior can be observed in thermometric tasks, when we want to infer the temperature of a bosonic bath by the same interaction with a probe. Even if the thermalized states corresponding to different temperatures can be distinguished, there is an advantage when measuring the probe at earlier times [16-18]. Interestingly enough, the statistics tagging setting and the thermometric setting also share another common feature: In both schemes the input states of the probe which ensure optimal performances correspond to energy eigenstates of its local Hamiltonian, with quantum coherence playing no fundamental role in the procedure (see, however, Ref. [8]). In an effort to check the generality of these observations (i.e., the optimality of using nonequilibrium observation times $t$ and energy diagonal input states of the probe), here we cast the problem studied in Ref. [15] in a more complex framework by looking at the discrimination between two alternative thermal baths models which differ both in terms of their statistical properties and in terms of their associated temperatures. The analysis relies on information-theoretic quantities which admit clear operational interpretations in quantum metrology [19-22]. In particular, the minimization of the Helstrom probability of error [23] enables us to confirm that also for the generalized statistics tagging scenario we address here, optimal discrimination performances are obtained by monitoring the probe at times where it is in a nonequilibrium configuration. However, in this case it turns out that such a result strongly relies on the possibility of exploiting coherence in the input states of $A$. Indeed, when restricting the study to initial configurations 
of the probe with no coherence among the eigenstates of the system local Hamiltonian, we can exhibit explicit examples of the model parameters for which the best discrimination conditions are only met at equilibrium.

The paper is organized as follows. In Sec. II we introduce the model and present the figure of merit we are going to use in our analysis. Section III contains the main results of the paper, discussing the role of coherent energy terms in the input state of the probe as well as the fact that nonequilibrium detection times are not always optimal if one restricts the analysis to initial configurations which are diagonal in the energy eigenbasis. In Sec. IV the previous concepts are illustrated in a linear-optical simulator [24,25]. The simulation allows us to mimic two different dissipative channels for a two-level system [26]. In the typical tagging scenario, in which we have no a priori knowledge of which one of the channels is acting on the probe, we perform a set of measurements on the system and we reconstruct the original hypothesis via suitable statistical inference. In particular, we rely on a Bayesian technique [27] for constructing the error probabilities and providing a connection of the latter with the theoretical figures mentioned above. A summary and conclusions are presented in Sec. V. Technical material is presented in the Appendixes.

\section{MODEL}

The model we study can be schematized as follows. At time $t=0$ a two-level (qubit) quantum probe $A$ is prepared in some fiduciary initial density operator $\hat{\rho}(0)$ and let to interact for some time $t$ with a partially unknown environment $E$ that can be of two types: bosonic at temperature $1 / \beta_{b}$ or fermionic at temperature $1 / \beta_{f}$ (the values $1 / \beta_{b}$ and $1 / \beta_{f}$ being assigned $a$ priori). As in Ref. [15], we will attempt to discriminate between the two alternatives by only performing measurements on the reduced final state $\hat{\rho}(t)$ of $A$, which hence encodes all the information about the nature of $E$ one can access. This allows us to describe the whole scheme as a standard hypothesis testing problem [23] where one has to determine whether $\hat{\rho}(t)$ corresponds to the density matrix $\hat{\rho}_{b}(t)$ of $A$, which one would have obtained by evolving $\hat{\rho}(0)$ under the influence of the bosonic bath of temperature $1 / \beta_{b}$, or to $\hat{\rho}_{f}(t)$, which instead one would have obtained by evolving the same $\hat{\rho}(0)$ under the influence of the fermionic bath of temperature $1 / \beta_{f}$. To quantify our ability in discriminating between these scenarios we can then use the Helstrom error probability (HEP) functional

$$
H\left[\hat{\rho}_{b}(t), \hat{\rho}_{f}(t)\right]:=\frac{1}{2}-\frac{1}{4}\left\|\hat{\rho}_{b}(t)-\hat{\rho}_{f}(t)\right\|_{1},
$$

with $\|\cdots\|_{1}$ being the trace-norm symbol. This quantity, bounded within $[0,1 / 2]$, provides the smallest probability of error one can get by optimizing over all possible measurements performed on a single copy of $\hat{\rho}(t)$ [23]. Accordingly, having $H\left[\hat{\rho}_{b}(t), \hat{\rho}_{f}(t)\right]=0$ corresponds to perfect distinguishable configurations, while having $H\left[\hat{\rho}_{b}(t), \hat{\rho}_{f}(t)\right]=1 / 2$ corresponds to absolutely indistinguishable configurations.

In order to get an analytical expression for (1) we assign $\hat{\rho}_{b}(t)$ and $\hat{\rho}_{f}(t)$ in terms of two independent GoriniKossakowski-Sudarshan-Lindblad master equations for $A$ obtained under standard weak-coupling system-bath assumptions [1,2]. Assuming the free Hamiltonian of $A$ to be

$$
\mathcal{H}_{0}=\frac{\omega_{0}}{2} \hat{\sigma}_{z}
$$

we move into the interaction picture to write the master equations as $[15,28,29]$

$$
\dot{\hat{\rho}}_{q}(t)=\gamma\left[1+s_{q} \mathcal{N}_{q}\left(\beta_{q}\right)\right] \mathcal{D}_{\hat{\sigma}_{-}}\left[\hat{\rho}_{q}(t)\right]+\gamma \mathcal{N}_{q}\left(\beta_{q}\right) \mathcal{D}_{\hat{\sigma}_{+}}\left[\hat{\rho}_{q}(t)\right],
$$

the index $q=f, b$ referring to the two hypothetical initial configurations of the bath. In the above expression $s_{q}=+1$ $(-1)$ for $q=b(f), \gamma$ is the inverse time constant associated with each elementary excitation-deexcitation process,

$$
\mathcal{D}_{\hat{\sigma}_{ \pm}}[\cdots]:=\hat{\sigma}_{ \pm}[\cdots] \hat{\sigma}_{ \pm}^{\dagger}-\frac{\hat{\sigma}_{ \pm}^{\dagger} \hat{\sigma}_{ \pm}[\cdots]+[\cdots] \hat{\sigma}_{ \pm}^{\dagger} \hat{\sigma}_{ \pm}}{2}
$$

represent the Lindblad dissipators associated, respectively, with the system ladder operators $\hat{\sigma}_{-}=|0\rangle\langle 1|$ and $\hat{\sigma}_{+}=$ $|1\rangle\langle 0|(|0\rangle$ and $|1\rangle$ representing, respectively, the ground and excited states of $A$ ), and

$$
\mathcal{N}_{q}\left(\beta_{q}\right):=\frac{1}{e^{\beta_{q} \omega}-s_{q}}
$$

is the Bose-Einstein (Fermi-Dirac) distribution for $q=b(f)$, with $\omega$ an effective energy parameter $[3,29]$ that contains a contribution from the bare energy $\omega_{0}$ of $A$ and from the chemical potential of the baths. ${ }^{1}$ Introducing the Pauli vector operator $\hat{\vec{\sigma}}:=\left(\hat{\sigma}_{x}, \hat{\sigma}_{y}, \hat{\sigma}_{z}\right)$ and writing the density matrix of the system in the Bloch vector formalism $\hat{\rho}_{q}(t)=\frac{1+\hat{\sigma} \cdot \vec{a}^{(q)}(t)}{2}$, Eq. (3) can then be conveniently cast in the form

$$
\begin{aligned}
& \dot{a}_{z}^{(q)}(t)=-\gamma_{q} a_{z}^{(q)}(t)-\xi_{q}, \\
& \dot{a}_{x}^{(q)}(t)=-\frac{\gamma_{q}}{2} a_{x}^{(q)}(t), \\
& \dot{a}_{y}^{(q)}(t)=-\frac{\gamma_{q}}{2} a_{y}^{(q)}(t),
\end{aligned}
$$

where now

$$
\begin{aligned}
& \gamma_{b}:=\gamma \operatorname{coth}\left(\beta_{b} \omega / 2\right), \quad \gamma_{f}:=\gamma, \\
& \xi_{b}:=\gamma, \quad \xi_{f}:=\gamma \tanh \left(\beta_{f} \omega / 2\right),
\end{aligned}
$$

showing that in the case of equal temperatures, the evolution occurs at faster scales for the bosonic bath scenario. Explicit integration of (6) leads finally to the solution

$$
\begin{aligned}
& a_{z}^{(q)}(t)=e^{-\gamma_{q} t}\left(a_{z}(0)-a_{z}^{(q)}(\infty)\right)+a_{z}^{(q)}(\infty), \\
& a_{x}^{(q)}(t)=e^{-\gamma_{q} t / 2} a_{x}(0), \\
& a_{y}^{(q)}(t)=e^{-\gamma_{q} t / 2} a_{y}(0),
\end{aligned}
$$

with $a_{x, y, z}(0)$ the Cartesian components of the Bloch vector associated with the input state $\hat{\rho}(0)$ of $A$ and

$$
a_{z}^{(q)}(\infty)=-\tanh \left(\beta_{q} \omega / 2\right)
$$

defining the equilibrium (thermal) configuration of the system [of course $a_{x}^{(q)}(\infty)=a_{y}^{(q)}(\infty)=0$ ]. Notice that the third

\footnotetext{
${ }^{1}$ We suppose $\omega$ to be the same for $b$ and $f$. When the chemical potential is different between the fermionic and bosonic cases, we can opportunely redefine $\beta_{f}$ and $\beta_{b}$ to preserve Eq. (5).
} 
component of the Bloch vector decays with a characteristic time scale $1 / \gamma_{q}$, while the one associated with the other two components is twice as long (see, e.g., Ref. [3], p. 149).

\section{DISCRIMINATION PERFORMANCES}

Using the fact that the trace norm of the difference between $\hat{\rho}_{b}(t)$ and $\hat{\rho}_{f}(t)$ is just given by the Cartesian distance $\left\|\vec{a}_{b}(t)-\vec{a}_{f}(t)\right\|_{2}$ of the associated three-dimensional Bloch vectors, from (8) it follows that Eq. (1) can be expressed as

$$
\begin{aligned}
H & {\left[\hat{\rho}_{b}(t), \hat{\rho}_{f}(t)\right] } \\
& =\frac{1}{2}-\frac{1}{4}\left\{\left[\left(e^{-\gamma_{f} t}-e^{-\gamma_{b} t}\right) a_{z}(0)+a_{z}^{(f)}(\infty)\left(1-e^{-\gamma_{f} t}\right)\right.\right.
\end{aligned}
$$

$$
\begin{aligned}
& \left.-a_{z}^{(b)}(\infty)\left(1-e^{-\gamma_{b} t}\right)\right]^{2} \\
& \left.+\left(e^{-\gamma_{f} t / 2}-e^{-\gamma_{b} t / 2}\right)^{2}\left[|\vec{a}(0)|^{2}-a_{z}^{2}(0)\right]\right\}^{1 / 2} .
\end{aligned}
$$

A close inspection reveals that all pure input states $\hat{\rho}(0)$ with the same initial value of $a_{z}(0)$ achieve the same performance [this simply follows from the symmetry of Eq. (6) around the $z$ axis]. Furthermore, and most importantly, for all assigned values of $t$ and $a_{z}(0)$, one may notice that the associated HEP can be reduced by setting the length of $\vec{a}(0)$ at its maximum 1 , i.e., imposing the initial state of the probe to be pure. In other words, mixedness in the input state is always detrimental, implying that, in order to find the best input configuration, it is sufficient to restrict the search to the set of pure states. This leads to

$$
\begin{aligned}
\left.H\left[\hat{\rho}_{b}(t), \hat{\rho}_{f}(t)\right]\right|_{\text {pure }}= & H_{p}\left[t ; a_{z}(0)\right]:=\frac{1}{2}-\frac{1}{4}\left\{\left[\left(e^{-\gamma_{f} t}-e^{-\gamma_{b} t}\right) a_{z}(0)+a_{z}^{(f)}(\infty)\left(1-e^{-\gamma_{f} t}\right)-a_{z}^{(b)}(\infty)\left(1-e^{-\gamma_{b} t}\right)\right]^{2}\right. \\
& \left.+\left[e^{-\gamma_{f} t / 2}-e^{-\gamma_{b} t / 2}\right]^{2}\left[1-a_{z}^{2}(0)\right]\right\}^{1 / 2}
\end{aligned}
$$

which only depends on the $z$ component $a_{z}(0) \in[-1,1]$ of the unit vector $\vec{a}(0)$. It is worth recalling that setting $a_{z}(0)=1$ $\left[a_{z}(0)=-1\right]$ corresponds to initializing $A$ in the excited state $|1\rangle$ (ground state $|0\rangle$ ) of its local Hamiltonian. In contrast, in the pure case scenario we are facing in Eq. (11), the condition $\left|a_{z}(0)\right|<1$ identifies input states of the probe which are proper superpositions of the energy eigenstates of the model. Our next goal is to minimize $H_{p}\left[t ; a_{z}(0)\right]$ with respect to all possible choices of $a_{z}(0)$ and of the evolution time $t$, for given values of the temperatures $1 / \beta_{f}$ and $1 / \beta_{b}$. Before doing so, however, we find it useful to consider first what happens when $a_{z}(0)=1$, a choice that is known to provide the best discriminating strength for statistical tagging under an equal bath temperature assumption (i.e., $\beta_{f}=\beta_{b}$ ) [15] and for thermometry [16].

\section{A. Input excited state}

Setting $a_{z}(0)=1$, i.e., assuming $A$ to be initialized in the excited state $|1\rangle$ of the model, Eq. (11) reduces to

$$
\begin{aligned}
H_{p}(t ; 1)= & \frac{1}{2}-\frac{1}{4} \mid e^{-\gamma_{f} t}-e^{-\gamma_{b} t}+a_{z}^{(f)}(\infty)\left(1-e^{-\gamma_{f} t}\right) \\
& -a_{z}^{(b)}(\infty)\left(1-e^{-\gamma_{b} t}\right) \mid,
\end{aligned}
$$

which we minimize numerically with respect to $t$ as a function of $\beta_{f}$ and $\beta_{b}$. The optimal times $\bar{t}$ we obtain and the corresponding values of $H_{p}(\bar{t} ; 1)$ are reported in Figs. 1(a) and 1(b), respectively. The plot reveals an asymmetry: For $\beta_{b} \geqslant \beta_{f}$ (fermionic bath hotter than bosonic bath) the best discrimination is still attained at finite time $(\bar{t}<\infty)$, where $A$ has not achieved full thermalization and is hence in a nonequilibrium configuration in line with the findings of Ref. [15]; in contrast, for $\beta_{b}<\beta_{f}$ (fermionic bath cooler than bosonic bath) it can be more advantageous to discriminate between the two channels by exploiting the steady state properties $(\bar{t}=\infty)$. This happens above the critical curve that defines the discontinuity in the contour plot of Fig. 1(a). An analytical treatment of this transition is given in Appendix $\mathrm{A}$, from which it results that, expressed in the $x=\tanh \left(\beta_{f} \omega / 2\right)$ and $y=\tanh \left(\beta_{b} \omega / 2\right)$ coordinates of Fig. 1, such critical curve is identified by solving the set of transcendental equations

$$
\begin{array}{r}
\left(2-e^{-\tau}\right)(1+x)-\left(2-e^{-\frac{\tau}{y}}\right)(1+y)=0, \\
e^{-\tau}(1+x)-e^{-\frac{\tau}{y}} y^{-1}(1+y)=0,
\end{array}
$$

with $\tau \geqslant 0$. We remark that the core of the above observation remains unchanged when we evaluate the discrimination efficiency of the process adopting different figures of merit. For instance, in Figs. 1(c) and 1(d) we focus on the Chernoff quantity $[30,31]$

$$
Q\left[\hat{\rho}_{b}(t), \hat{\rho}_{f}(t)\right]:=\min _{r \in[0,1]} \operatorname{Tr}\left[\hat{\rho}_{b}^{r}(t) \hat{\rho}_{f}^{1-r}(t)\right],
$$

which via the inequality

$$
H\left[\hat{\rho}_{b}^{\otimes N}(t), \hat{\rho}_{f}^{\otimes N}(t)\right] \leqslant \frac{Q\left[\hat{\rho}_{b}(t), \hat{\rho}_{f}(t)\right]^{N}}{2}
$$

gives a bound to the asymptotic rate of HEP computed in the case when one has the possibility of extracting information from $N$ identical copies of the final state of $A$. The optimal values of $\bar{t}$ obtained by numerically minimizing (14) when initializing $A$ in the excited state $|1\rangle$ are presented in Fig. 1(c), which exhibits a critical trade-off analogous to the one observed in Fig. 1(a): If we restrict the analysis to the case where $A$ is set in the excited state, there are configurations of the model where the optimal discrimination efficiency is attained only by letting the system reach its equilibrium configuration.

\section{B. Optimal input states of the probe}

In this section we exploit the full domain of possibilities offered by the model, minimizing the HEP value (11) not just with respect to $t$, but also with respect to the full domain of $a_{z}(0)$, hence including the possibility of using input states of $A$ which explicitly exhibit coherence superpositions among the excited and ground states of the model. An indication that such special states could be of some help in improving the performance of the scheme follows by observing that for 


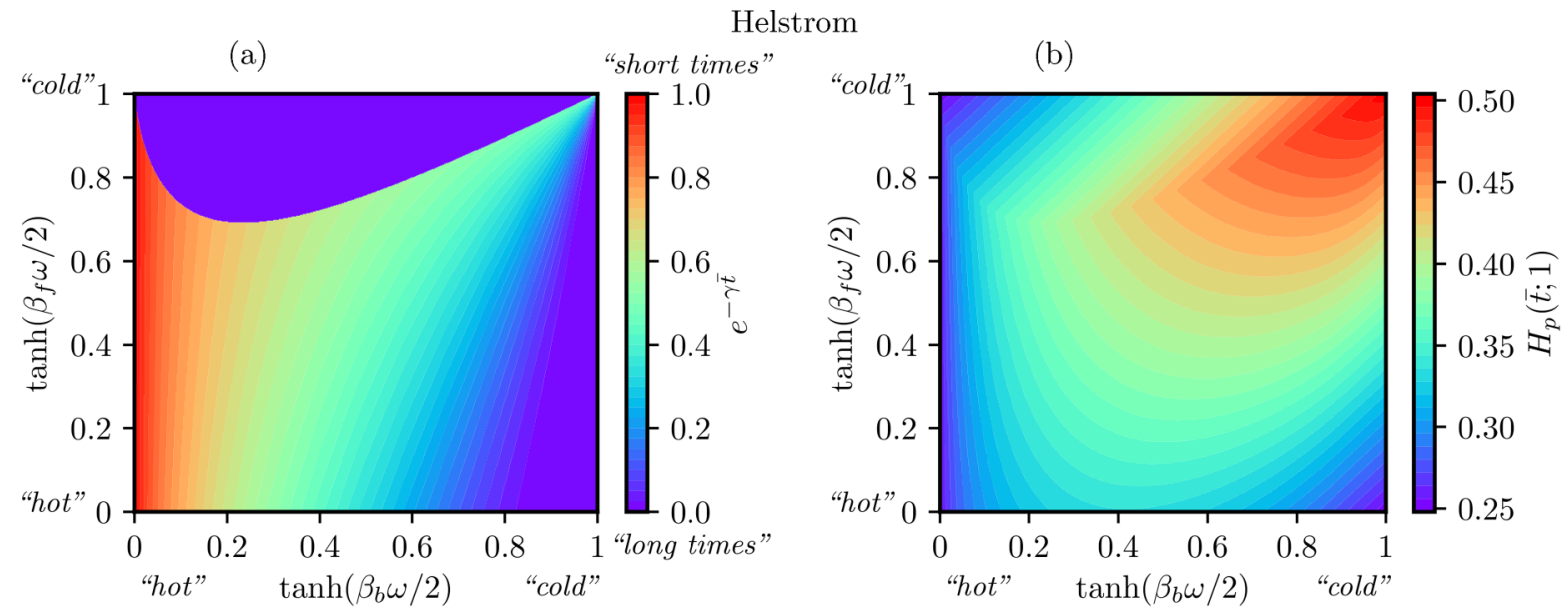

Chernoff
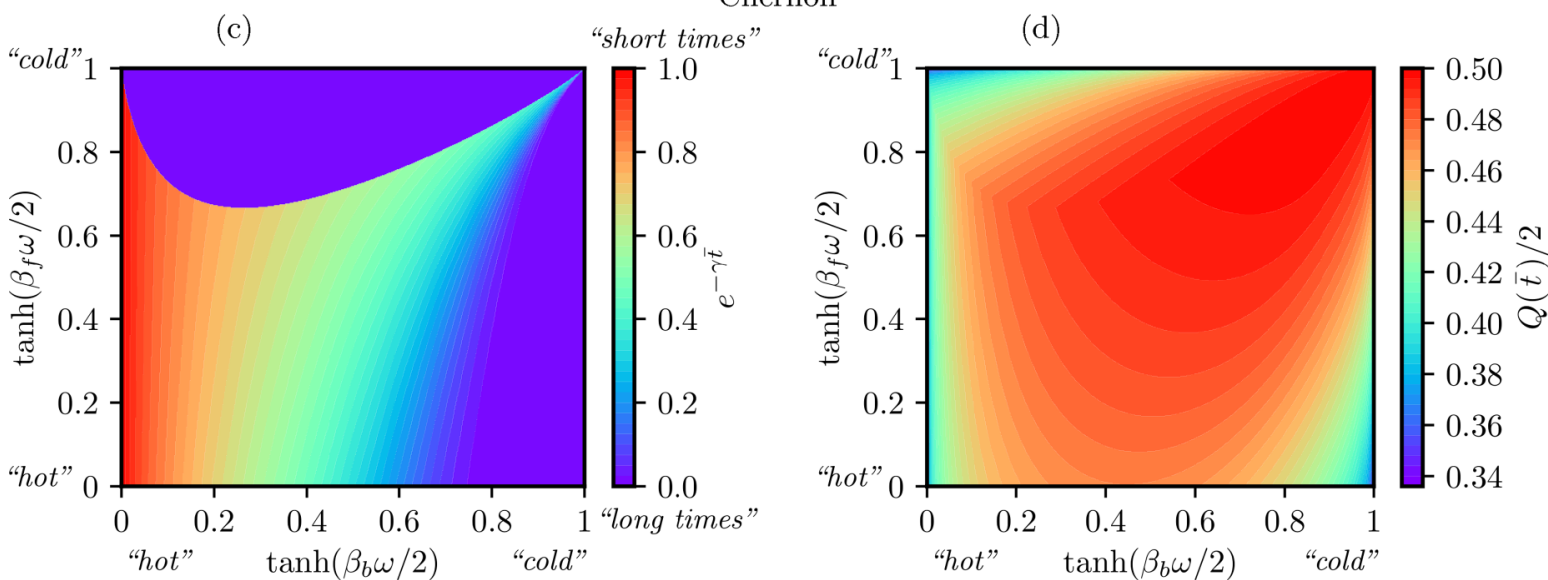

FIG. 1. (a) Study of the optimal measurement time $\bar{t}$ minimizing the Helstrom error probability $H_{p}(t ; 1)$ of Eq. (12) associated with the excited input state of the probe $A$, i.e., $a_{z}(0)=1$, as a function of the bosonic and fermionic bath inverse temperatures $\beta_{b}$ and $\beta_{f}$, respectively. For convenience, we used the parametrizations $e^{-\gamma \bar{t}}$ for $\bar{t}$ and $\tanh \left(\beta_{q} \omega / 2\right)$ for $\beta_{q}$ (see the plot labels). The discontinuity in the contour plot is the boundary above which the discrimination is optimal only if performed on the steady state of the probe $\left(\bar{t}=\infty\right.$, i.e., $\left.e^{-\gamma \bar{t}}=0\right)$, the same holding for the pathological case $\beta_{b}=\infty\left[\tanh \left(\beta_{b} \omega / 2\right)=1\right]$. For all the other values of the parameters $\beta_{b}$ and $\beta_{f}$ the optimal time is finite $\left(\bar{t}<\infty\right.$, i.e., $\left.e^{-\gamma \bar{t}}>0\right)$. (b) Corresponding Helstrom probability of error $H_{p}(t ; 1)$ evaluated at $t=\bar{t}$. (c) and (d) Same calculation as in (a) and (b), respectively, but using the Chernoff quantity (14) instead of the Helstrom error probability.

$\left|a_{z}(0)\right|<1$ it is not possible to find times $t>0$ such that $H_{p}\left[t ; a_{z}(0)\right]$ reaches the worst case value of $1 / 2$ corresponding to an absolute impossibility of distinguishing between the two bath scenarios. This implies that coherent energy input states ensure a nontrivial susceptibility of the probe for all choices of $t$, something that, on the contrary, is not generally granted by setting $a_{z}(0)= \pm 1$, which, as discussed in Appendix B 1, allows for crossing points between the trajectories of $\hat{\rho}_{b}(t)$ and $\hat{\rho}_{f}(t)$. Values of $\left|a_{z}(0)\right|<1$ can however do much more than this and in some regimes they also give the absolute best performance we can aim for. The details of this analysis are provided in Appendix B 2, while in Fig. 2 we illustrate the optimization of the HEP $H_{p}\left[t ; a_{z}(0)\right]$ over time and input state of the probe, as a function of the bath inverse temperatures $\beta_{f}$ and $\beta_{b}$

The first thing to be noticed is that now, at variance with the input excited state case discussed in Sec. III A, the optimal times $\bar{t}$ are always finite apart from the asymptotic regimes where the bosonic temperature converges to zero (i.e., $\beta_{b} \rightarrow \infty$ ) [compare Fig. 2(a) with Fig. 1(a)]. This shows that optimality of nonequilibrium probing times is fully restored once we do not restrict the probe input state to specific conditions. Put differently, allowing coherence in the initial state implies that the quantity $\left\{1 / 2-H_{p}\left[\bar{t} ; \bar{a}_{z}(0)\right]\right\} / \bar{t}$, which we can interpret as an information rate, is never exactly nullified $\left[\bar{a}_{z}(0)\right.$ being the optimal choice for $\left.a_{z}(0)\right]$. Second, Fig. 2(b) reveals that, while using energy eigenstates (either excited or ground states) of the probe as input is optimal for most of the choices of the system parameter setting, there is a nontrivial temperatures regime in which a coherent $\left[\left|a_{z}(0)\right|<1\right]$ initial preparation is fundamental to reach the best performance. More specifically, there is numerical evidence that whenever the fermionic bath is hotter than the bosonic one $\left(\beta_{b} \geqslant \beta_{f}\right)$, choosing the excited state of $A$ as input is still the right choice to provide optimal discrimination performances. The situation changes however if the fermionic bath is cooler than the bosonic one $\left(\beta_{b}<\beta_{f}\right)$ : Here the optimal input choice depends on the specific values of the temperatures and in particular for sufficiently large $\beta_{f}$ coherent energy states can dominate (notice also that, for small values of $\beta_{b}$, the optimal input can 

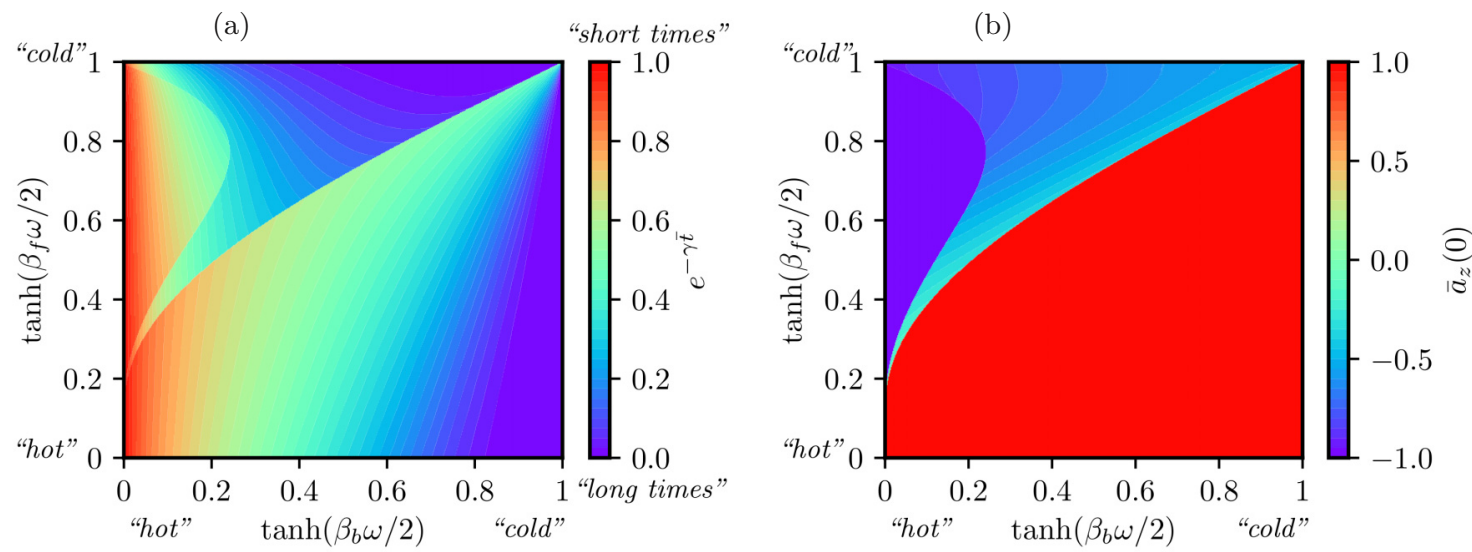

(c)

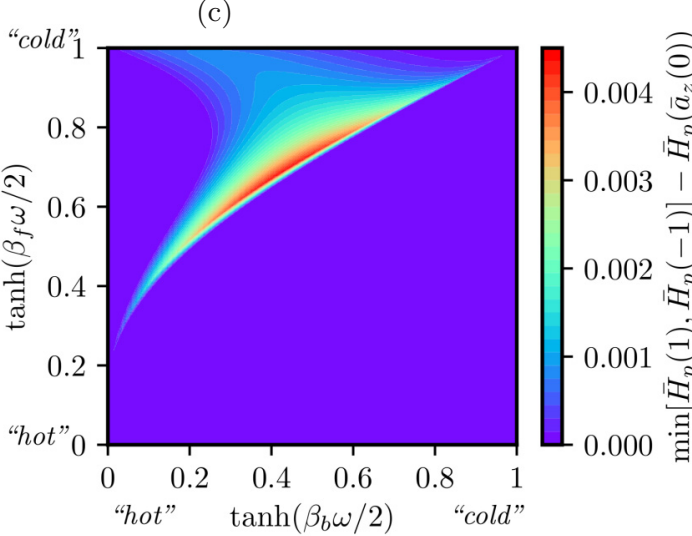

(d)

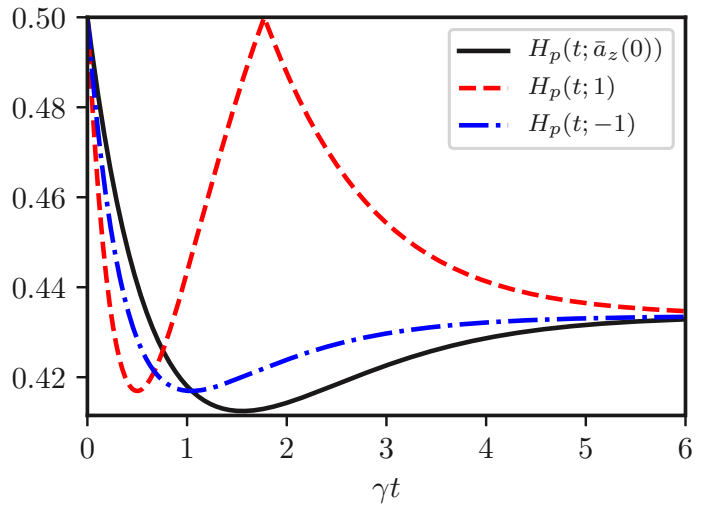

FIG. 2. (a)-(c) Optimization of the HEP $H_{p}\left[t ; a_{z}(0)\right]$ of Eq. (11) both over time and over the input state of the probe, the latter being a generic pure state with a certain value $a_{z}(0)$ of the $z$ component of the Bloch vector. We report the following contour plots with respect to the bath inverse temperatures $\beta_{f}$ and $\beta_{b}$. The minimum $\bar{H}_{p}\left[\bar{a}_{z}(0)\right]$ of the HEP is achieved (a) at a certain time $\bar{t}$ and (b) for an optimal value $\bar{a}_{z}(0)$ of $a_{z}(0)$. (c) Advantage coming from allowing coherent superpositions, where we show the gap between the (generally overestimated) quantity obtained by restricting the analysis only to $a_{z}(0) \in\{1,-1\}$ and the optimal value $\bar{H}_{p}\left[\bar{a}_{z}(0)\right]$. Notice that for $\beta_{f}, \beta_{b}$, and $\bar{t}$ we used the convenient parametrizations indicated in the plot labels. (d) Dynamical evolution of $H_{p}\left[t ; a_{z}(0)\right]$ for a case $\left[\tanh \left(\beta_{f} \omega / 2\right) \approx 0.68\right.$ and $\left.\tanh \left(\beta_{b} \omega / 2\right) \approx 0.41\right]$ in which coherent superpositions $\left[\bar{a}_{z}(0) \approx-0.42\right]$ give better performances than the energy eigenstates $\left[a_{z}(0) \in\{1,-1\}\right]$ as input of the probe. Notice that at $t \approx 1.8 \gamma^{-1}$ the HEP associated with excited state reaches the worst case value $1 / 2$, indicating zero susceptibility of the probe.

be the ground state of $A$ ). These facts are also highlighted in Fig. 2(c), in which we show the gap between the minimum of $H_{p}\left[t ; a_{z}(0)\right]$ obtained by restricting the optimization only to $a_{z}(0)=1$ and $a_{z}(0)=-1$ and the optimal value $\bar{H}_{p}\left[\bar{a}_{z}(0)\right]$ obtained by allowing also energy coherent preparations.

In Fig. 2(d) we present as an example the temporal evolution of the HEP for a specific choice of the temperatures that admits as optimal the value $\bar{a}_{z}(0) \approx-0.42$, which identifies a coherent superposition of energy eigenstates. In such a plot we show $H_{p}\left[t ; \bar{a}_{z}(0)\right]$ in addition to the HEP values $H_{p}(t ;-1)$ and $H_{p}(t ; 1)$ associated with the ground and excited input states of $A$, respectively. Notice that while for small $t$, $H_{p}(t ;-1)$ and $H_{p}(t ; 1)$ perform better than $H_{p}\left[t ; \bar{a}_{z}(0)\right]$, in the long run the latter gives the lowest HEP values and leads to the identification of the optimal time as $\bar{t} \approx 1.6 \gamma^{-1}$ [see Eq. (B14) for more on this]. Notice also that at $t \approx 1.8 \gamma^{-1}$, we have $H_{p}(t ; 1)=1 / 2$, indicating that at this special time the probe intialized in the excited state loses all its ability in discriminating between the two alternative hypotheses: On the contrary, as anticipated in the introductory paragraphs of the section, $H_{p}\left[t ; \bar{a}_{z}(0)\right]$ remains strictly below the $1 / 2$ value for all positive $t$.

\section{DISCRIMINATION EXPERIMENT IN AN OPTICAL SIMULATOR}

We can illustrate these concepts in a simulated thermalization, carried out with a pair of qubits; the necessary gate is implemented by means of optical elements and coincidence counts. The setup, illustrated in Fig. 3, follows closely our previous work in Ref. [24]. We stress that our simulator cannot replicate directly the bosonic/fermionic nature of the bath; the control parameters are exclusively the decay rates $\gamma_{f}$ or $\gamma_{b}$ in Eq. (6) and the population of the final thermal state. In this respect, our implementation is a synthesis of the output state. Therefore, we focus on the information content of the probe rather than the interaction process.

We consider a two-level system initialized in the excited state as the input probe. The expectation values of $\hat{\sigma}_{z}$ measured as a function of the normalized time $\tau=\gamma t$ are shown in 

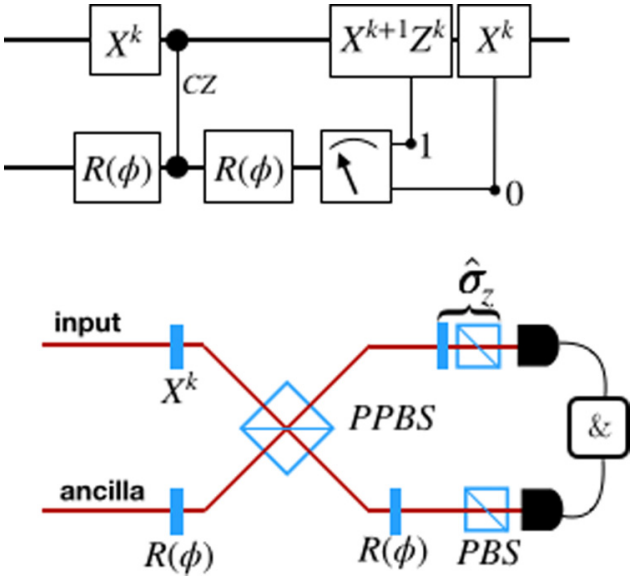

FIG. 3. Linear optical simulator. The state at time $\tau$ can be simulated by mixing with weights $w_{k}$ the actions of two channels $(k=0$ and $k=1$ ), associated with excitation and deexcitation processes, for a given rotation $R(\phi)$ (upper panel). By tuning $w_{k}$ and $\phi$ evolutions at different temperatures and at variable times are simulated [24,25]. This is implemented in a linear-optical setup, based on polarization coding on two photons from a down-conversion source (lower panel). Single-qubit operations are either implemented by half waveplates or in postprocessing of the data. The input state is fixed in the horizontal polarization. Due to the use of a single partially polarizing beam splitter (PPBS), there is a different transmission probability for the horizontal and vertical components, which is compensated by biasing the second $R(\phi)$ rotation [32]. Further, the weights $w_{k}$ have to be modified accordingly.

Fig. 4 for different inverse temperatures $\beta \omega=0.5,1,2$, taken to be equal for fermionic and bosonic baths. The two different curves in each panel illustrate how the decay rate of the probe state gets modified by the two different statistics.

In a discrimination experiment, the sought outcome is a binary decision on which one of the two hypotheses gives a closer description of the data [33-38]. These will be obtained as outcomes of a suitable observable, selected according to the initial state and the measurement time. For our choice of initial state, this observable always coincides with $\hat{\sigma}_{z}$. In many different (and independent) runs of the experiment, one collects $N_{0}$ events for the eigenvalue -1 and $N_{1}$ events for the eigenvalue +1 of $\hat{\sigma}_{z}$. Since the probabilities of obtaining either result on a single copy are $P_{i}^{(q)}=\left[1+(-1)^{i+1}\left\langle\hat{\sigma}_{z}\right\rangle^{(q)}(t)\right] / 2$, where the value of $\left\langle\hat{\sigma}_{z}\right\rangle^{(q)}$ is the expectation value predicted by the experiment, the composite probability is $\mathbf{P}=\left(P_{0}^{(q)}\right)^{N_{0}}\left(P_{1}^{(q)}\right)^{N_{1}}$.

Clearly, the probability $\mathbf{P}$ depends on the bath statistics and temperature through the expectation value $\left\langle\hat{\sigma}_{z}\right\rangle$. We can thus interpret $\mathbf{P}$ as a conditioned probability $\mathbf{P}\left(N_{0}, N_{1} \mid X\right)$ of the whole experimental run, given the condition $X$ of the bath. Invoking Bayes's theorem, this is written

$$
\mathbf{P}\left(X \mid N_{0}, N_{1}\right)=\frac{1}{\mathcal{N}} \mathbf{P}\left(N_{0}, N_{1} \mid X\right) P(X),
$$

where $\mathcal{N}$ is a normalization constant and $P(X)$ is the a priori probability which we take to be flat $P(b)=P(f)=1 / 2$. The decision criterion is that when $P\left(b \mid N_{0}, N_{1}\right)>P\left(f \mid N_{0}, N_{1}\right)$, the bath is identified as bosonic with inverse temperature $\beta_{b}$ and otherwise as fermionic with inverse temperature $\beta_{f}$.

Taking inspiration from Refs. [23,39], we can quantify the ideal discrimination error as

$$
\delta=\frac{1}{2}\left[P\left(b, \beta_{b} \mid N_{0}^{f, \beta_{f}}, N_{1}^{f, \beta_{f}}\right)+P\left(f, \beta_{f} \mid N_{0}^{b, \beta_{b}}, N_{1}^{b, \beta_{b}}\right)\right],
$$

where we have set $N_{i}^{q, \beta_{q}}=P_{i}^{(q)} N(N=10$ or 100). This provides an indication of the expected performance of the Bayesian technique in light of the imperfections of our experiment. On the other hand, this figure of merit does not capture the impact of statistical fluctuations with respect to the mean values.

The first case we analyze is that of statistical tagging $\beta_{b}=\beta_{f}$, for which optimal discrimination necessarily occurs at finite times. In Fig. 5 we show the behavior of $\delta$ for $\beta_{b}=\beta_{f}$. We notice that the small discrepancies observed with respect to the theory do not affect the estimation significantly. It appears evident how, for high temperatures, the choice of a preferable discrimination time becomes less strict with increasing copies $N$. On the other hand, the proximity of the two curves in Fig. 4(c) is reflected in the fact that at low temperatures more copies are needed for a fully reliable discrimination.

Concerning the more general scenario of different temperatures and statistics, we have evaluated $\delta$ for all permutations of $\beta \omega=0.5,1,2$ and for $N=10,100$ in the same ideal limit as above. The results are shown in Fig. 6. Notably, for $\beta_{b}<\beta_{f}$ there is a special time instant where the discrimination is impossible, in analogy to what we obtained for the HEP [see
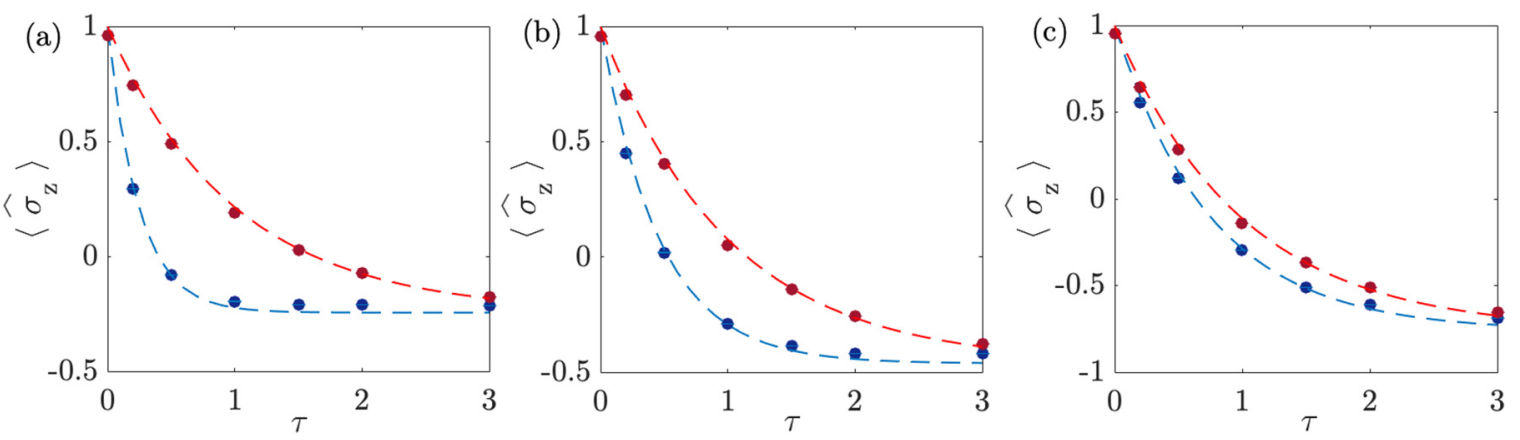

FIG. 4. Simulated thermalization dynamics of the probe initialized in the excited state. The behavior of the expectation value $\left\langle\hat{\sigma}_{z}\right\rangle$ as a function of the time $\tau=\gamma t$ is reported for $(\omega \equiv 1)$ (a) $\beta_{b}=\beta_{f}=0.5$, (b) $\beta_{b}=\beta_{f}=1$, and (c) $\beta_{b}=\beta_{f}=2$. Blue (red) experimental points refer to the bosonic (fermionic) statistics of the bath. Error bars are smaller than the size of the points. 

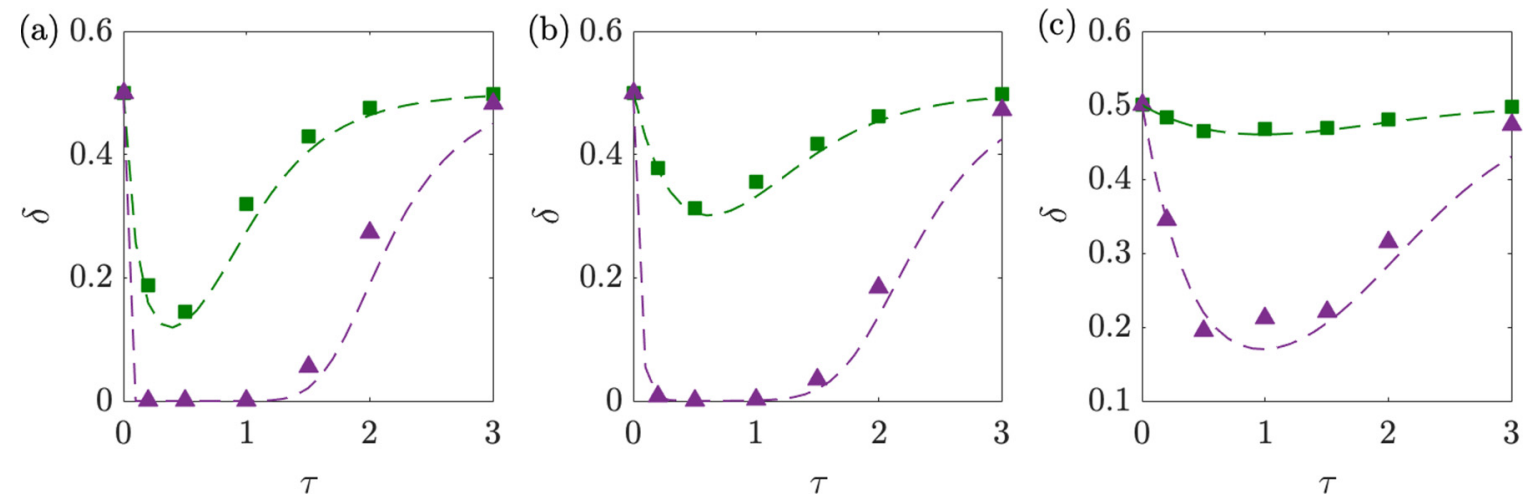

FIG. 5. Discrimination errors for statistical tagging. The error $\delta$ of Eq. (17) is evaluated for (a) $\beta_{b}=\beta_{f}=0.5$, (b) $\beta_{b}=\beta_{f}=1$, and (c) $\beta_{b}=\beta_{f}=2(\omega \equiv 1)$. In all panels, points are evaluations of the expected errors based on the experimental probabilities and the curves are the ideal cases for $N=10$ (green squares) or $N=100$ (purple triangles). The probe was initialized in the excited state.

Fig. 2(d)]. This can be observed in Figs. 6(a), 6(b), and 6(d), where, in contrast to the other panels, $\delta$ takes the value $1 / 2$ at an intermediate time. We report in Fig. 7 contour plots showing the calculation of the optimal measurement time and of the corresponding minimized error probability when using the Bayesian method in the ideal situation. Consistently, the results mimic the ones obtained via Helstrom and Chernoff approaches (see Fig. 1).

Inspection of Figs. 6 and 7 suggests that working with $N=$ 10 copies of the state would not lead to satisfactory tagging capabilities, even without taking into account the relevance of statistical fluctuations. In the same ideal limit, $N=100$ copies should be sufficient for nearly unambiguous tagging around ideal time. In these conditions, it is important to quantify how the deviations of $N_{0}$ and $N_{1}$ from their mean values affect the tagging.

We then carry out the actual discrimination protocol as follows. We generate, based on the experimental values of $P_{0}$ and $P_{1}$, a vector of $N=100$ outcomes $0,1 .^{2}$ This is a reliable evaluation of our experimental conditions, as the data are marginally affected by systematic errors such as dark

\footnotetext{
${ }^{2}$ This is achieved by generating a random number $r$ uniformly between 0 and 1 . If $r<P_{0}$, then $N_{0}$ is incremented by one unit (starting from $N_{0}=N_{1}=0$ ); otherwise $N_{1}$ is incremented.
}

(a)

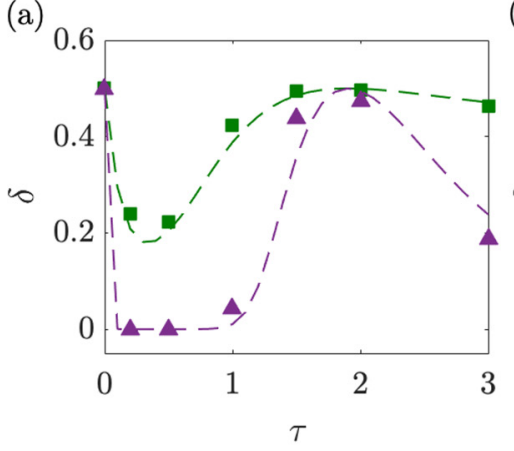

(d)

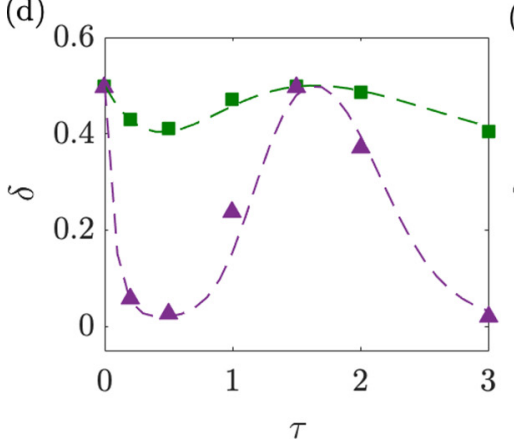

(b)

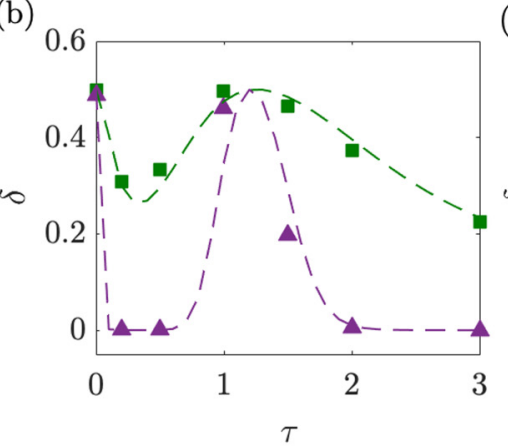

(c)

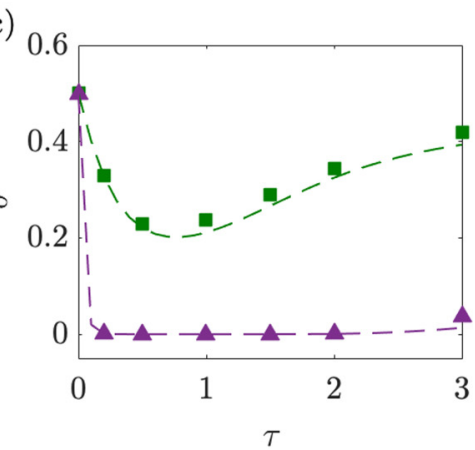

(e)

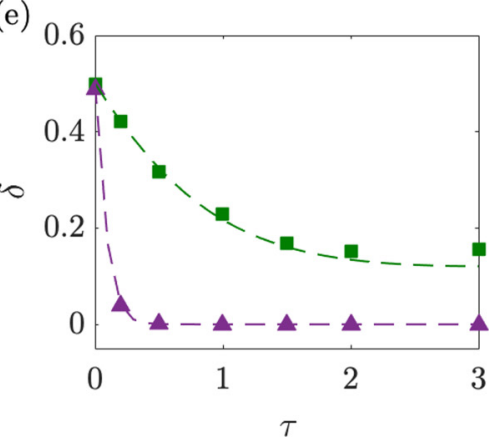

(f)

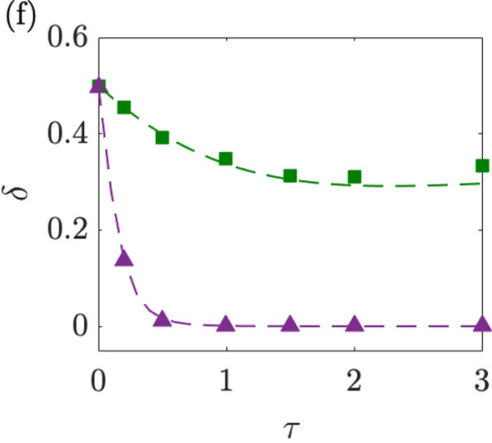

FIG. 6. Discrimination errors for statistical tagging. The error $\delta$ of Eq. (17) is evaluated for (a) $\beta_{b}=0.5$ and $\beta_{f}=1$, (b) $\beta_{b}=0.5$ and $\beta_{f}=2$, (c) $\beta_{b}=1$ and $\beta_{f}=0.5$, (d) $\beta_{b}=1$ and $\beta_{f}=2$, (e) $\beta_{b}=2$ and $\beta_{f}=0.5$, and (f) $\beta_{b}=2$ and $\beta_{f}=1(\omega \equiv 1)$. In all panels, points are evaluations of the expected errors based on the experimental probabilities and the curves are the ideal cases for $N=10$ (green squares) or $N=100$ (purple triangles). The probe was initialized in the excited state. 


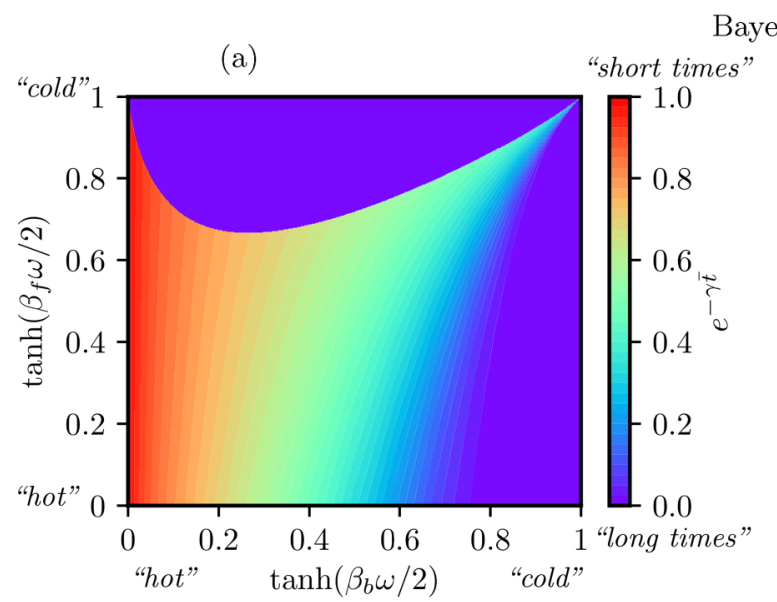

Bayes $(\mathrm{N}=10)$
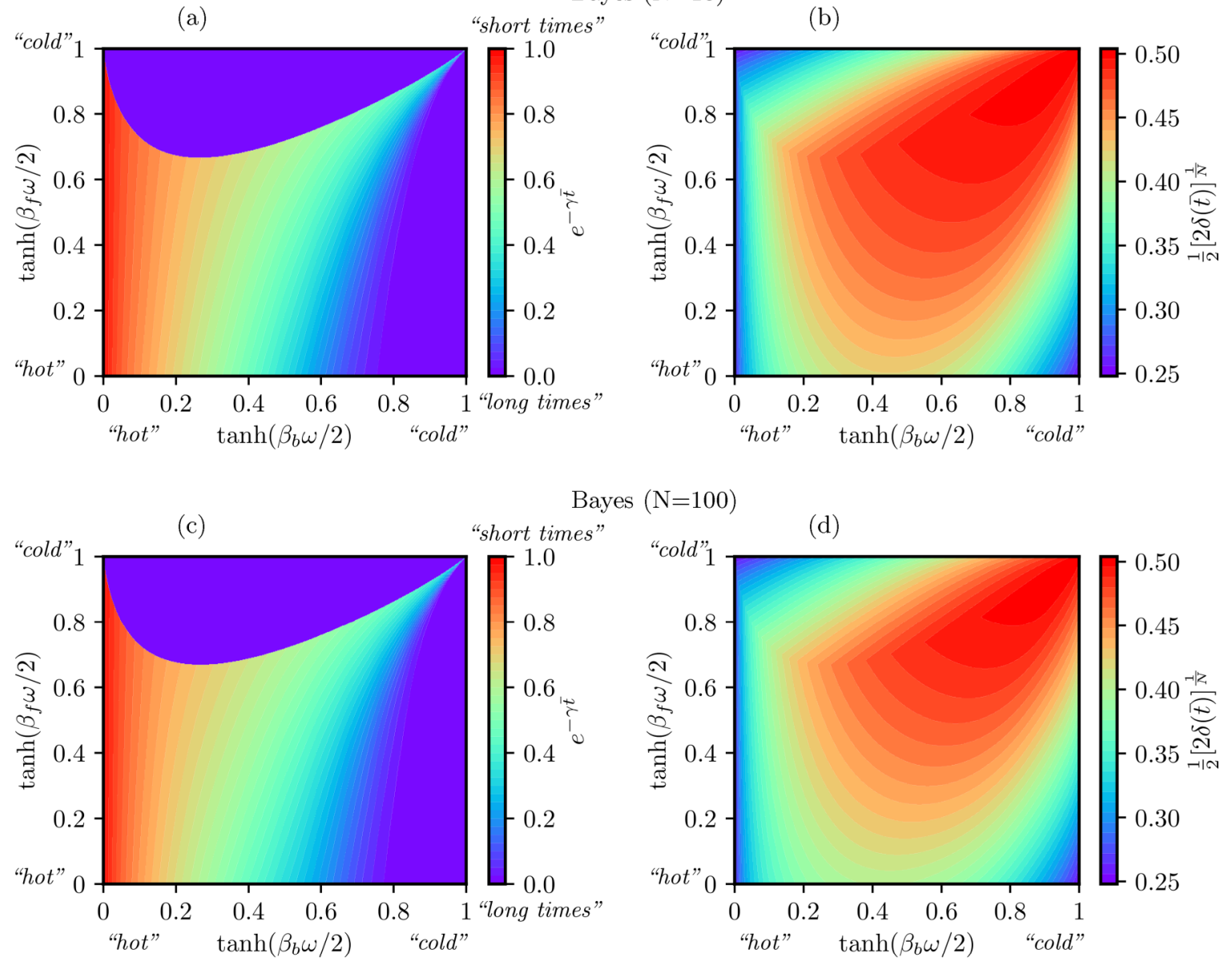

Bayes $(\mathrm{N}=100)$

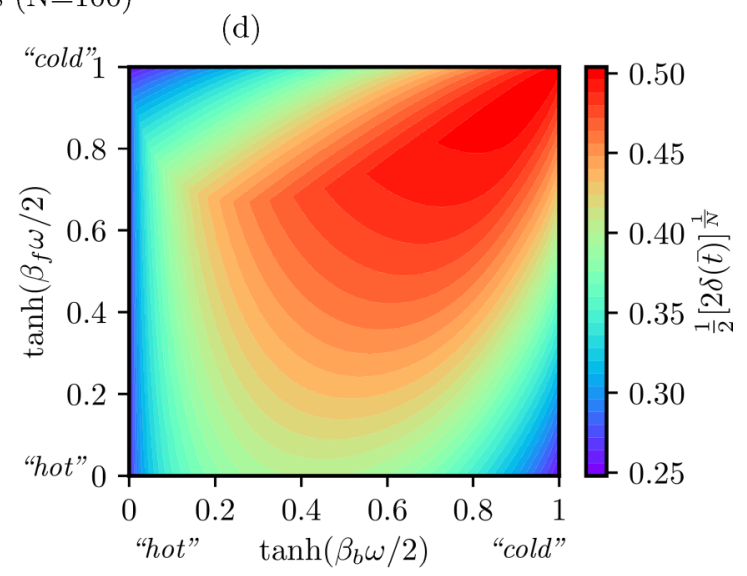

FIG. 7. Same study as in Fig. 1 but for the error probability $\delta$ of Eq. (17) based on the Bayesian approach for (a) and (b) $N=10$ and (c) and (d) $N=100$. To allow a direct comparison for different $N$ and with the figures of merit of Fig. 1, we report instead of $\delta$ its rescaled version $1 / 2[2 \delta]^{1 / N}$. We remark that as for Fig. 1, the probe was initialized in the excited state.

counts and we are considering samples much smaller than those collected to estimate $P_{0}$ and $P_{1}$ in the calibration step. The results are reported, for a vector of $N=100$ generated outcomes, in the histograms of Fig. 8 for different choices of scenarios, considering both instances in which the probe is associated with a bosonic or a fermionic bath, in accordance with the fact that the error $\delta$ is symmetrized.

For each simulated time $\tau$ we indicate with different colors the fraction of events in which the bath has been correctly identified (blue) or mistaken (red) by following the Bayesian decision rule explained above, now with the actual values of $N_{0}$ and $N_{1}$, rather than their expected ones. The observed behaviors qualitatively mirror the errors in Figs. 5 and 6. This implies that, although $N_{0}$ and $N_{1}$ are now random variables, this stochastic element does not impact considerably the discrimination capability at the optimal time.

\section{CONCLUSION}

Statistical tagging [15] and, more generally, bath discrimination are simple yet insightful instances of the possibility of indirectly probing an environment [4-14,40]. In this setting, information about the bath structure are retrieved via measurements on a quantum probe which has interacted with the bath until a selected measurement time $\bar{t}$. This approach reveals how different properties of the bath affect the nature of the optimal discrimination procedures. This is clear in the tagging context presented here: A thermal bath has unknown statistics, fermionic or bosonic, that we want to guess, with the additional information of knowing the respective temperatures, $1 / \beta_{f}$ and $1 / \beta_{b}$, associated with the two bath instances. Here the quantum nature of the problem is manifested both in the statistical properties of the bath and in the coherence of the single-qubit probe. As intuitively expected, the best discrimination capability can only be attained by enforcing the probe initial state to be pure. For input energy eigenstates, our inspection has revealed a transition between temperature regimes in which either equilibrium, $\bar{t} \rightarrow \infty$, or nonequilibrium states, $\bar{t}<\infty$, are optimal. Such behavior has been illustrated both theoretically and in a linear-optical simulation. However, only the inclusion of input states with quantum coherence allows one to reach the highest possible discrimination capability and also implies that nonequilibrium measurement conditions are generally optimal, breaking in this way the aforementioned transition.

Extensions of this work may concern baths with richer features, such as very large baths presenting squeezing or, to the other extreme, small environments, entailing more involved 
(a)

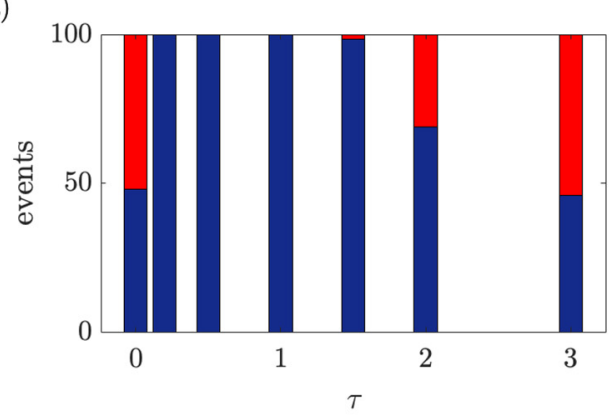

(c)

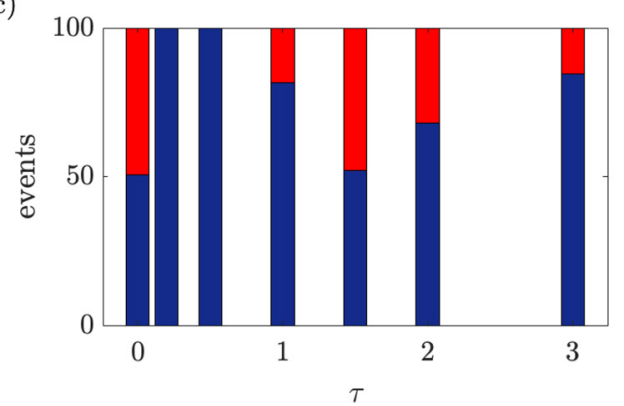

(b)

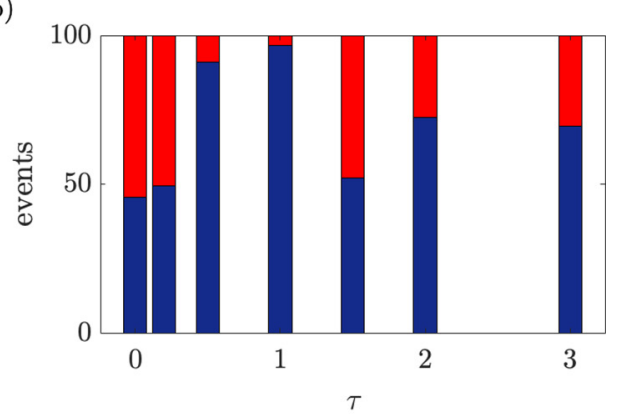

(d)

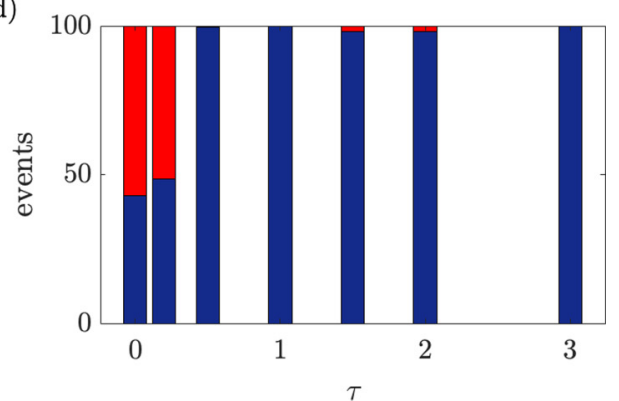

FIG. 8. Bayesian bath discrimination. The histograms report the events correctly identified in blue (lower part of the histogram bars) and the incorrect ones in red (upper part of the bar). The discrimination tasks are (a) statistical tagging with $\beta_{b}=\beta_{f}=0.5$, (b) statistical tagging with $\beta_{b}=\beta_{f}=2$, (c) bath discrimination with $\beta_{b}=0.5$ and $\beta_{f}=1$, and (d) bath discrimination with $\beta_{b}=2$ and $\beta_{f}=1(\omega \equiv 1)$. The probe was initialized in the excited state.

treatments. There we can expect coherence properties of the probe to become even more relevant, thus adding richness and complexity to the observable phenomenology.

Note added. Recently, we became aware of a related work by Mancino et al. [41] also dealing with metrological tasks by means of indirectly measuring environments via quantum probes.

\section{ACKNOWLEDGMENTS}

V.G. acknowledges support from MIUR via PRIN 2017 (Progetto di Ricerca di Interesse Nazionale): project QUSHIP (2017SRNBRK).

\section{APPENDIX A: EXCITED INPUT STATE}

Equation (1) clearly shows that the minimal values of HEP are achieved when $\left\|\hat{\rho}_{b}(t)-\hat{\rho}_{f}(t)\right\|_{1}$ becomes maximum. From Eq. (12) it follows that for the case of an excited input state, i.e., for $a_{z}(0)=1$, this quantity can be expressed as

$$
\begin{aligned}
\left\|\hat{\rho}_{b}(t)-\hat{\rho}_{f}(t)\right\|_{1}= & D(t, x, y):=\mid\left(1-e^{-\gamma t}\right)(1+x) \\
& -\left(1-e^{-\gamma t / y}\right)(1+y) \mid,
\end{aligned}
$$

where, for ease of notation, we have introduced

$$
\begin{aligned}
x & :=-a_{z}^{(f)}(\infty)=\tanh \left(\beta_{f} \omega / 2\right), \\
y & :=-a_{z}^{(b)}(\infty)=\tanh \left(\beta_{b} \omega / 2\right),
\end{aligned}
$$

and made explicit $\gamma_{f}=\gamma$ and $\gamma_{b}=\gamma / y$. Studying Eq. (A1) as a function of $t$, we can infer which instant is optimal to perform a single measurement for discriminating between the two hypotheses.
First, we notice that $D(t, x, y)$ is nullified at $t=0$ (obviously) and at most at another point, since by solving $D(t, x, y)=0$ we have

$$
\frac{1+x}{1+y}=\frac{1-e^{-\gamma t / y}}{1-e^{-\gamma t}} .
$$

The unicity of the solution can be argued using the monotonicity of the right-hand side of Eq. (A3). Notice that the other solution (at $t=0$ ) cannot be obtained from Eq. (A3) since we divided by $1-e^{-\gamma t}$, which is nullified in that case. The first derivative of $D(t, x, y)$ with respect to time reads

$$
\begin{aligned}
D^{\prime}(t)= & \gamma \operatorname{sgn}\left[\left(1-e^{-\gamma t}\right)(1+x)-\left(1-e^{-\gamma t / y}\right)(1+y)\right] \\
& \times\left[(1+x) e^{-\gamma t}-(1+y) y^{-1} e^{-\gamma t / y}\right],
\end{aligned}
$$

which clearly is nullified in the long time limit $\gamma t \rightarrow \infty$. To find other zeros of $D^{\prime}$ we have to solve the equation

$$
\frac{1+x}{1+y^{-1}}=e^{-\gamma t\left(y^{-1}-1\right)},
$$

which can have at most one solution since the right-hand side is a strictly decreasing function. In addition, it is possible to prove that, calling $t_{1}$ and $t_{2}$ the zeros at finite time of $D(t, x, y)$ and of its first derivative, respectively, we have $t_{1} \geqslant t_{2}$. Indeed, they satisfy the two equations (A3) and (A5), from which we derive

$$
\frac{e^{-\gamma t_{2}\left(y^{-1}-1\right)}}{y}=\frac{1-e^{-\gamma t_{1} / y}}{1-e^{-\gamma t_{1}}}
$$

Now we can use the inequality $\frac{e^{-\gamma t_{2}\left(y^{-1}-1\right)}}{y} \leqslant \frac{1-e^{-\gamma t_{2} / y}}{1-e^{-\gamma t_{2}}}$, from which $t_{1} \geqslant t_{2}$ can be argued using the decreasing properties of both sides of Eq. (A6). 
As a last step we want to study the behavior of the zeros in the parameters $x$ and $y$. It is straightforward to verify that Eq. (A3) has no solutions if $x<y$, since there is no crossing between the bosonic and fermionic evolutions in this case. Notice that, following the definition of $x$ and $y$, this last condition is equivalent to requiring the inverse temperature $\beta_{f}$ in the fermionic case to be lower than the one in the bosonic case $\beta_{b}$. Notice instead that Eq. (A5) is always nullified once, independently of the values of $x$ and $y$. In conclusion, we have two possible qualitative trends for the trace norms (A1).

(i) If $\beta_{f}<\beta_{b}, D(t)$ starts from 0 and is never nullified again. The derivative of $D$ is zero once in such a way that there is one single maximum. This case includes the analysis done in [15], in which $\beta_{f}=\beta_{b}$ was considered.

(ii) If $\beta_{f}>\beta_{b}, D(t)$ reaches a maximum in $t_{2}$ and then decreases to a point $t_{1}$ in which it attains the value 0 . After $t_{1}, D(t)$ starts increasing again and remains monotonic when going to infinity.

\section{Analysis of the critical point}

For the sake of characterizing the optimal measurements, we should find the maxima of the trace norm studied in the preceding section. In the case $\beta_{f}<\beta_{b}$ there is only one maximum and the measurement should be clearly done in the instant of time associated with that maximum. In the case $\beta_{f}>\beta_{b}$ the intermediate maximum could be either greater or lesser than the value attained by $D(t)$ at infinitely long times. In the following we will show that, again, dependent on the values assumed by the inverse temperatures $\beta_{f}$ and $\beta_{b}$, either one or the other strategy can be the best one.

To give a clear formulation to this question from a formal point of view, let us define the function

$$
g(t, x, y)=D(t)-\lim _{t \rightarrow \infty} D(t) .
$$

The zeros of the function defined above correspond to the points at which $D(t)$ attains the same value as it does at $t=\infty$. Thus, if the equation $g(t, x, y)=0$ has no solutions, the absolute maximum is clearly located at $t \rightarrow \infty$. Otherwise, given the properties of $D(t)$ enumerated in the preceding section, the function $g(t, x, y)$ can have at most two zeros, depending on the values of $x$ and $y$. In this last case, the maximum is not located at $t=\infty$, since this last point is equal to at most two other values that the function $D(t)$ attains at finite time. It is also understood that the points at which $g(t, x, y)$ has a unique zero (which from now on will be referred to as critical) are the ones at which $D(t)$ has two absolute maxima (identical in value). If we fix the value of $y$ to some value $\bar{y}$, we have that at a critical point the solution $x_{c}(t, \bar{y})$ of $g(t, x, \bar{y})=0$ must be such that $\frac{\partial}{\partial t} x(t, \bar{y})=0$ evaluated at the critical point [this last property is derived from the regularity of $g$ and the definition of critical point, in which the equation $g(t, x, y)=0$ passes from having zero to two solutions]. We can then derive, using the definition of $g$, the set of equations for the critical point

$$
\begin{aligned}
\left(2-e^{-\gamma t}\right)(1+x)-\left(2-e^{-\gamma t / \bar{y}}\right)(1+\bar{y}) & =0, \\
e^{-\gamma t}(1+x)-e^{-\gamma t / \bar{y}} \bar{y}^{-1}(1+\bar{y}) & =0,
\end{aligned}
$$

which replacing $\gamma t$ with $\tau$ gives Eq. (13). For instance, choosing $\bar{y}=1 / 2$, we have $x_{c}=\frac{2 \sqrt{2}-1}{\sqrt{2}+1}$ and $\gamma t_{c}=\ln \left(\frac{\sqrt{2}+1}{\sqrt{2}}\right)$. Then, if we choose $x<x_{c}$ the better strategy is to measure at finite time, while if $x>x_{c}$ the measurement at the steady state is the optimal one [see Fig. 1(a)].

\section{APPENDIX B: DISCRIMINATION WITH GENERIC PURE INPUT STATES}

Here we proceed with an analytical analysis of the HEP functional $H_{p}\left[t ; a_{z}(0)\right]$ defined in Eq. (11).

\section{Loss of susceptibility under noncoherent inputs}

The worst discrimination scenario is attained when HEP reaches its maximum value $1 / 2$. When this happens the probability of error is maximum and we cannot recover information on the nature of the bath from the state of $A$. From Eq. (1) this happens when $\left\|\hat{\rho}_{b}(t)-\hat{\rho}_{f}(t)\right\|_{1}=0$, i.e., when the two trajectories intercept. From Eq. (11) we observe that this can only occur when

$$
\begin{aligned}
& \left(e^{-\gamma_{f} t / 2}-e^{-\gamma_{b} t / 2}\right)^{2}\left[1-a_{z}^{2}(0)\right]=0, \\
& \left(e^{-\gamma_{f} t}-e^{-\gamma_{b} t}\right) a_{z}(0)+a_{z}^{(f)}(\infty)\left(1-e^{-\gamma_{f} t}\right) \\
& \quad-a_{z}^{(b)}(\infty)\left(1-e^{-\gamma_{b} t}\right)=0 .
\end{aligned}
$$

However, setting $\left|a_{z}(0)\right|<1$, i.e., allowing the input state of $A$ to be a nontrivial superposition of the energy eigenstates, corresponds to

$$
\begin{aligned}
e^{-\gamma_{f} t / 2} & =e^{-\gamma_{b} t / 2}, \\
{\left[a_{z}^{(f)}(\infty)-a_{z}^{(b)}(\infty)\right]\left(1-e^{-\gamma_{b} t}\right) } & =0,
\end{aligned}
$$

which can only be fulfilled for $t=\infty$ and $\beta_{f}=\beta_{b}$. On the contrary, setting $a_{z}(0)= \pm 1$ (i.e., forcing the probe to be in one of the two eigenstates of the system), the system (B1) reduces to a single equation

$$
\begin{aligned}
& \pm\left(e^{-\gamma_{f} t}-e^{-\gamma_{b} t}\right)+a_{z}^{(f)}(\infty)\left(1-e^{-\gamma_{f} t}\right) \\
& \quad-a_{z}^{(b)}(\infty)\left(1-e^{-\gamma_{b} t}\right)=0,
\end{aligned}
$$

which, depending on the specific values of $\beta_{b}$ and $\beta_{f}$, may allow for nontrivial $t>0$ solutions, i.e., indicating a loss of susceptibility of the probe.

\section{Full optimization}

We are interested in determining the minimum value of Eq. (11) with respect to all possible inputs (i.e., all possible choices of $\left.a_{z}(0) \in[-1,1]\right)$ and all possible times $t \geqslant 0$. According to (1) this is formally equivalent to determining the maximum of $\left\|\hat{\rho}_{b}(t)-\hat{\rho}_{f}(t)\right\|_{1}$, which in this case is given by the function

$$
\begin{aligned}
\| \hat{\rho}_{b}(t) & -\hat{\rho}_{f}(t) \|_{1} \\
= & D\left[t ; a_{z}(0)\right]:=\left\{\left[\left(e^{-\gamma_{f} t}-e^{-\gamma_{b} t}\right) a_{z}(0)+a_{z}^{(f)}(\infty)\right.\right. \\
& \left.\times\left(1-e^{-\gamma_{f} t}\right)-a_{z}^{(b)}(\infty)\left(1-e^{-\gamma_{b} t}\right)\right]^{2} \\
& \left.+\left(e^{-\gamma_{f} t / 2}-e^{-\gamma_{b} t / 2}\right)^{2}\left[1-a_{z}^{2}(0)\right]\right\}^{1 / 2} .
\end{aligned}
$$


The best way to approach the problem seems to first optimize with respect to $a_{z}(0)$ and then maximize with respect to $t$. Again, we call $\left[\bar{t} ; \bar{a}_{z}(0)\right]$ the point where the maximum value of $D^{2}\left[t ; a_{z}(0)\right]$ is attained.

Let us fix $t$ and rewrite $D^{2}\left[t ; a_{z}(0)\right]$ as a parabola in $a_{z}(0)$, $D^{2}\left[t ; a_{z}(0)\right]=f_{-}^{2}\left(f_{+}^{2}-1\right) a_{z}^{2}(0)+2 A f_{-} f_{+} a_{z}(0)+f_{-}^{2}+A^{2}$,

with

$$
\begin{aligned}
f_{ \pm} & :=e^{-\gamma t / 2} \pm e^{-\gamma t /(2 y)}, \\
A & :=-x\left(1-e^{-\gamma t}\right)+y\left(1-e^{-\gamma t / y}\right),
\end{aligned}
$$

where we used (A2) to express the dependence upon $\beta_{b}$ and $\beta_{f}$. Since $a_{z}(0) \in[-1,1], \bar{a}_{z}(0)$ is either one of the extrema -1 and 1 or the abscissa of the vertex $V=A f_{+} /\left[f_{-}\left(1-f_{+}^{2}\right)\right]$ of the parabola (B5). The condition for the vertex to be the maximum is that the parabola is concave down and that the abscissa of the vertex falls strictly inside the interval ] $-1,1[$ :

$$
\left.\frac{f_{-}}{f_{+}}\left(1-f_{+}^{2}\right)>|A| \Leftrightarrow \bar{a}_{z}(0)=V \in\right]-1,1[.
$$

On the other hand, its violation imposes that the maximum is one of the extrema depending on the sign of $A$,

$$
\frac{f_{-}}{f_{+}}\left(1-f_{+}^{2}\right) \leqslant|A| \Leftrightarrow \bar{a}_{z}(0)=\operatorname{sgn}[A] .
$$

The equation above holds for $A \neq 0$; when $A=0$ the points $a_{z}(0)=1$ and $a_{z}(0)=-1$ are two equivalent maxima (still provided the function is concave up).

Eventually, we have to find the maximum among $D^{2}\left(t_{1} ; V_{t_{1}}\right), D^{2}\left(t_{2} ; 1\right)$, and $D^{2}\left(t_{2} ;-1\right)$ for all $t_{1}$ satisfying inequality (B7) and $t_{2}$ satisfying inequality (B8). The explicit values of the three quantities above can be computed from Eq. (B5) and read

$$
\begin{gathered}
D^{2}\left(t_{1} ; V_{t_{1}}\right)=\frac{f_{-}^{2}\left(t_{1}\right)\left[f_{+}^{2}\left(t_{1}\right)-1\right]-A^{2}\left(t_{1}\right)}{f_{+}^{2}\left(t_{1}\right)-1}, \\
D^{2}\left(t_{2} ; 1\right)=\left[f_{+}\left(t_{2}\right) f_{-}\left(t_{2}\right)+A\left(t_{2}\right)\right]^{2}, \\
D^{2}\left(t_{2} ;-1\right)=\left[f_{+}\left(t_{2}\right) f_{-}\left(t_{2}\right)-A\left(t_{2}\right)\right]^{2} .
\end{gathered}
$$

Such a maximization procedure yields the point $\left[\bar{t}, \bar{a}_{z}(0)\right]$ we were searching for fixed $x$ and $y$. However, notice that, in general (for both the case in which the concavity is up and the case in which it is down) the sign of $A$ determines the sign of $\bar{a}_{z}(0)$,

$$
\operatorname{sgn}\left[\bar{a}_{z}(0)\right]=\operatorname{sgn}[A]
$$

implying that in the region

$$
y \geqslant x \Rightarrow \bar{a}_{z}(0)>0 .
$$

Moreover, the condition (B7) cannot be satisfied for $t$ sufficiently close to 0 such that

$$
\frac{\gamma t / 2}{\ln \left(\frac{1}{1-\exp (-\gamma t / 2)}\right)} \leqslant y \Rightarrow \bar{a}_{z}(0)=\operatorname{sgn}[A] .
$$

This short time condition leads to the conclusion that the coherent advantage can occur only in a "long run" but still out of equilibrium, as previously illustrated in Fig. 2.
[1] G. Lindblad, On the generators of quantum dynamical semigroups, Commun. Math. Phys. 48, 119 (1976).

[2] V. Gorini, A. Kossakowski, and E. C. G. Sudarshan, Completely positive dynamical semigroups of $N$-level systems, J. Math. Phys. 17, 821 (1976).

[3] H.-P. Breuer and F. Petruccione, The Theory of Open Quantum Systems (Oxford University Press, Oxford, 2002).

[4] M. Brunelli, S. Olivares, and M. G. A. Paris, Qubit thermometry for micromechanical resonators, Phys. Rev. A 84, 032105 (2011).

[5] L. A. Correa, M. Mehboudi, G. Adesso, and A. Sanpera, Individual Quantum Probes for Optimal Thermometry, Phys. Rev. Lett. 114, 220405 (2015).

[6] A. De Pasquale, K. Yuasa, and V. Giovannetti, Estimating temperature via sequential measurements, Phys. Rev. A 96, 012316 (2017).

[7] S. Campbell, M. G. Genoni, and S. Deffner, Precision thermometry and the quantum speed limit, Quantum Sci. Technol. 3, 025002 (2018).

[8] A. H. Kiilerich, A. De Pasquale, and V. Giovannetti, Dynamical approach to ancilla-assisted quantum thermometry, Phys. Rev. A 98, 042124 (2018).
[9] M. Schlosshauer, A. P. Hines, and G. J. Milburn, Decoherence and dissipation of a quantum harmonic oscillator coupled to two-level systems, Phys. Rev. A 77, 022111 (2008).

[10] J. P. Pekola, S. Suomela, and Y. M. Galperin, Finite-size bath in qubit thermodynamics, J. Low Temp. Phys. 184, 1015 (2016).

[11] S. Gröblacher, A. Trubarov, N. Prigge, G. D. Cole, M. Aspelmeyer, and J. Eisert, Observation of non-Markovian micromechanical Brownian motion, Nat. Commun. 6, 7606 (2015).

[12] N. V. Prokof'ev and P. C. E. Stamp, Theory of the spin bath, Rep. Prog. Phys. 63, 669 (2000).

[13] A. De Pasquale, D. Rossini, R. Fazio, and V. Giovannetti, Local quantum thermal susceptibility, Nat. Commun. 7, 12782 (2016).

[14] C. Benedetti, F. Salari Sehdaran, M. H. Zandi, and M. G. A. Paris, Quantum probes for the cutoff frequency of Ohmic environments, Phys. Rev. A 97, 012126 (2018).

[15] D. Farina, V. Cavina, and V. Giovannetti, Quantum bath statistics tagging, Phys. Rev. A 100, 042327 (2019).

[16] S. Jevtic, D. Newman, T. Rudolph, and T. M. Stace, Singlequbit thermometry, Phys. Rev. A 91, 012331 (2015). 
[17] W. K. Tham, H. Ferretti, A. V. Sadashivan, and A. M. Steinberg, Simulating and optimising quantum thermometry using single photons, Sci. Rep. 6, 38822 (2016).

[18] L. Mancino, M. Sbroscia, I. Gianani, E. Roccia, and M. Barbieri, Quantum Simulation of Single-Qubit Thermometry using Linear Optics, Phys. Rev. Lett. 118, 130502 (2017).

[19] V. Giovannetti, S. Lloyd, and L. Maccone, Quantum Metrology, Phys. Rev. Lett. 96, 010401 (2006).

[20] M. G. Paris, Quantum estimation for quantum technology, Int. J. Quantum Inf. 7, 125 (2009).

[21] V. Giovannetti, S. Lloyd, and L. Maccone, Advances in quantum metrology, Nat. Photon. 5, 222 (2011).

[22] S. Pirandola, B. R. Bardhan, T. Gehring, C. Weedbrook, and S. Lloyd, Advances in photonic quantum sensing, Nat. Photon. 12, 724 (2018).

[23] C. W. Helstrom, Quantum Detection and Estimation Theory (Academic, New York, 1976).

[24] L. Mancino, V. Cavina, A. De Pasquale, M. Sbroscia, R. I. Booth, E. Roccia, I. Gianani, V. Giovannetti, and M. Barbieri, Geometrical Bounds on Irreversibility in Open Quantum Systems, Phys. Rev. Lett. 121, 160602 (2018).

[25] V. Cavina, L. Mancino, A. De Pasquale, I. Gianani, M. Sbroscia, R. I. Booth, E. Roccia, R. Raimondi, V. Giovannetti, and M. Barbieri, Bridging thermodynamics and metrology in nonequilibrium quantum thermometry, Phys. Rev. A 98, 050101(R) (2018).

[26] H. Lu, C. Liu, D.-S. Wang, L.-K. Chen, Z.-D. Li, X.-C. Yao, L. Li, N.-L. Liu, C.-Z. Peng, B. C. Sanders, Y.-A. Chen, and J.-W. Pan, Experimental quantum channel simulation, Phys. Rev. A 95, 042310 (2017).

[27] M. G. Genoni, S. Olivares, D. Brivio, S. Cialdi, D. Cipriani, A. Santamato, S. Vezzoli, and M. G. A. Paris, Optical interferometry in the presence of large phase diffusion, Phys. Rev. A 85, 043817 (2012).

[28] D. Farina and V. Giovannetti, Open-quantum-system dynamics: Recovering positivity of the Redfield equation via the partial secular approximation, Phys. Rev. A 100, 012107 (2019).

[29] K. L. M. Esposito, R. Kawai, and C. Van den Broeck, Quantumdot Carnot engine at maximum power, Phys. Rev. E 81, 041106 (2010).

[30] K. M. R. Audenaert, J. Calsamiglia, R. Muñoz-Tapia, E. Bagan, L. Masanes, A. Acin, and F. Verstraete, Discriminating States:
The Quantum Chernoff Bound, Phys. Rev. Lett. 98, 160501 (2007).

[31] J. Calsamiglia, R. Muñoz-Tapia, L. Masanes, A. Acin, and E. Bagan, Quantum Chernoff bound as a measure of distinguishability between density matrices: Application to qubit and Gaussian states, Phys. Rev. A 77, 032311 (2008).

[32] B. P. Lanyon, T. J. Weinhold, N. K. Langford, M. Barbieri, D. F. V. James, A. Gilchrist, and A. G. White, Experimental Demonstration of a Compiled Version of Shor's Algorithm with Quantum Entanglement, Phys. Rev. Lett. 99, 250505 (2007).

[33] P. J. Mosley, S. Croke, I. A. Walmsley, and S. M. Barnett, Experimental Realization of Maximum Confidence Quantum State Discrimination for the Extraction of Quantum Information, Phys. Rev. Lett. 97, 193601 (2006).

[34] G. J. Pryde, J. L. O'Brien, A. G. White, and S. D. Bartlett, Demonstrating Superior Discrimination of Locally Prepared States using Nonlocal Measurements, Phys. Rev. Lett. 94, 220406 (2005)

[35] S. Slussarenko, M. M. Weston, J.-G. Li, N. Campbell, H. M. Wiseman, and G. J. Pryde, Quantum State Discrimination using the Minimum Average Number of Copies, Phys. Rev. Lett. 118 030502 (2017)

[36] M. Bina, A. Allevi, M. Bondani, and S. Olivares, Homodynelike detection for coherent state-discrimination in the presence of phase noise, Opt. Express 25, 10685 (2017).

[37] M. T. DiMario, E. Carrasco, R. A. Jackson, and F. E. Becerra, Implementation of a single-shot receiver for quaternary phaseshift keyed coherent states, J. Opt. Soc. Am. B 35, 568 (2018).

[38] F. E. Becerra, J. Fan, and A. Migdall, Implementation of generalized quantum measurements for unambiguous discrimination of multiple non-orthogonal coherent states, Nat. Commun. 4 2028 (2013).

[39] M. A. Nielsen and I. L. Chuang, Quantum Computation and Quantum Information (Cambridge University Press, Cambridge, 2000)

[40] M. Mehboudi, A. Lampo, C. Charalambous, L. A. Correa M. A. García-March, and M. Lewenstein, Using Polarons for Sub-nK Quantum Nondemolition Thermometry in a BoseEinstein Condensate, Phys. Rev. Lett. 122, 030403 (2019).

[41] L. Mancino et al., Nonequilibrium readiness and precision of Gaussian quantum thermometers, Phys. Rev. Res. 2, 033498 (2020). 